\title{
Hyponatremia as a prognostic factor in non-small cell lung cancer: a systematic review and meta-analysis
}

\author{
Birgitte Sandfeld-Paulsen ${ }^{1}$, Ninna Aggerholm-Pedersen ${ }^{2}$, Anne Winther-Larsen ${ }^{1,3}$ \\ ${ }^{1}$ Department of Clinical Biochemistry, Aarhus University Hospital, Aarhus, Denmark; ${ }^{2}$ Department of Clinical Oncology, Aarhus University \\ Hospital, Aarhus, Denmark; ${ }^{3}$ Department of Clinical Biochemistry, Randers Regional Hospital, Randers, Denmark \\ Contributions: (I) Conception and design: B Sandfeld-Paulsen, A Winther-Larsen; (II) Administrative support: B Sandfeld-Paulsen, A Winther-Larsen; \\ (III) Provision of study materials or patients: B Sandfeld-Paulsen, A Winther-Larsen; (IV) Collection and assembly of data: B Sandfeld-Paulsen, \\ A Winther-Larsen; (V) Data analysis and interpretation: All authors; (VI) Manuscript writing: All authors; (VII) Final approval of manuscript: All \\ authors. \\ Correspondence to: Anne Winther-Larsen, MD, PhD. Department of Clinical Biochemistry, Randers Regional Hospital, Skovlyvej 15, 8930 Randers \\ NØ, Denmark. Email: anlarsen@rm.dk.
}

Background: Hyponatremia is a common electrolyte disorder in cancer patients and has been evaluated as a negative prognostic factor in several cancer types, especially in small cell lung cancer (SCLC). However, the prognostic value of hyponatremia is less studied in non-small cell lung cancer (NSCLC) patients. Therefore, we conducted a systematic review and meta-analysis to investigate the prognostic value of pretreatment hyponatremia in NSCLC patients.

Methods: The review was conducted in accordance with the Preferred Reporting Items for Systematic Reviews and Meta-Analyses (PRISMA) reporting checklist. The databases PubMed, Embase, Scopus, and Web of Science were searched on March 26th 2020 without time restrictions. The protocol was submitted to the PROSPERO database (ID 184612). Studies were included if they evaluated sodium level as a prognostic factor in NSCLC patients and contained original data published in English. Hazard ratios (HRs) for overall survival (OS) were pooled in a random-effects model.

Results: Of 10,888 identified titles, 14 articles were included in the review including a total of 4,364 NSCLC patients. In 13 of the 14 articles, hyponatremia was found to be significantly correlated to a shorter OS. Ten articles were included in the meta-analysis. Here, patients with hyponatremia had a significantly shorter OS compared with patients with normal sodium level \{pooled HR =2.02 [95\% confidence interval (CI): 1.65-2.47]\}. Two out of four studies found hyponatremia to be associated with a reduced progression free survival (PFS). Normalization of the sodium level during treatment was found to be associated with a prolonged PFS and OS in one study.

Conclusions: This meta-analysis indicates that hyponatremia is a relative common condition in NSCLC patients associated with an increased mortality. Hence, sodium level could be a useful biomarker for stratifying NSCLC patients and thereby for preparing individual treatment plans.

Keywords: Non-small cell lung cancer (NSCLC); sodium; hyponatremia; biomarkers; survival

Submitted Jul 21, 2020. Accepted for publication Nov 26, 2020.

doi: $10.21037 /$ tlcr-20-877

View this article at: http://dx.doi.org/10.21037/tlcr-20-877 


\section{Introduction}

Lung cancer is one of the most common cancers and the most common course of cancer death worldwide (1). Non-small cell lung cancer (NSCLC) accounts for approximately $85 \%$ of all lung cancer while small cell lung cancer (SCLC) comprises the remaining 15\%. In spite of great achievements in anticancer treatment of NSCLC patients in the last decades, the clinical outcome of the disease remains unsatisfactory with a 5-year survival rate less than $20 \%$ (2).

To improve the management and survival of NSCLC patients, estimation of a patient's prognosis is fundamental as it can guide clinicians to decide if anticancer therapy is likely to be beneficial for the patient. Moreover, it helps both the clinician and the patient to predict the natural history of the disease leading to more optimal treatment courses and planning of the end-of-life care. Therefore, identification of prognostic factors is essential. To date, disease stage is the most important identified prognostic factor in NSCLC patients together with performance status (PS), age, histology and sex (3). However, additional factors are wanted to guide the clinicians to perform more accurate estimations.

Hyponatremia is a common electrolyte disorder in cancer patients and has been evaluated as a negative prognostic factor of survival in several cancer types (4-6). It is defined as a sodium level below $135 \mathrm{mmol} / \mathrm{L}$ and further classified into three severities; mild hyponatremia (130-134 mmol/L), moderate hyponatremia $(125-129 \mathrm{mmol} / \mathrm{L})$ and severe hyponatremia $(<125 \mathrm{mmol} / \mathrm{L})$. Patients with hyponatremia are often asymptomatic or showing only a few unspecific symptoms like headache, fatigue, confusion, vomiting and hallucinations (6). The incidence is depended on the kind of malignancy as very different frequencies have been described in different cancer types $(5,7,8)$. In lung cancer, the incidence of hyponatremia is high in SCLC patients (9), and the influence of hyponatremia on survival has been intensively evaluated in these patients $(10,11)$. However, the prognostic value of hyponatremia is less studied in NSCLC patients, and studies have shown inconsistent results $(12,13)$. Therefore, we conducted a systematic review and a metaanalysis to investigate the prognostic value of pretreatment hyponatremia in NSCLC patients.

We present the following article in accordance with the Preferred Reporting Items for Systematic Reviews and Meta-Analyses (PRISMA) reporting checklist (14) (available at http://dx.doi.org/10.21037/tlcr-20-877).

\section{Methods}

\section{Data sources and search strings}

A comprehensive search was performed in PubMed, Embase, Scopus and Web of Science on the $26^{\text {th }}$ of March, 2020 with no time restriction. PubMed and Embase were filtered for English, Danish, Swedish and Norwegian only while Scopus and Web of Science was filtered for English only. In addition, PubMed and Embase were filtered for "not animals". The following search strings were used: PubMed: ("Lung Neoplasms"[Mesh] OR lung cancer OR lung carcinoma* OR lung neoplasm*) AND ("Hyponatremia"[Mesh] OR Hyponatremia* OR "Sodium"[Mesh] OR Sodium). Embase: ('lung tumor'/ exp OR (lung AND cancer) OR (lung AND carcinoma*) OR (lung AND neoplasm*) AND ('hyponatremia'/exp OR 'sodium'/exp OR sodium OR hyponatremia*). Scopus: ("lung carcinomas" OR "lung carcinoma" OR "lung cancer" OR "lung neoplasm") AND ("Hyponatremia" OR “sodium”). Web of Science: ("lung cancer" OR "lung tumor" OR "lung tumors" OR "lung carcinoma” OR "lung carcinomas" OR "lung neoplasm" OR "lung neoplasms") AND ("Hyponatremia" OR "sodium”).

\section{Study selection}

The inclusion criteria were as follows: (I) original data, (II) human studies, (III) patients with a pathological proven histology of NSCLC (IV) studies evaluating sodium level as a prognostic factor. Exclusion criteria were as follows: (I) language other than English, Danish, Swedish and Norwegian, (II) reviews, meta-analyses, guidelines, editorials, comments, case reports including fewer than five cases, letters to the editor or other communications without original data, (III) conference abstracts, (IV) animal or in vitro studies. When multiple publications using the same study population were identified or when the study populations overlapped, only the study including the largest number of NSCLC patients was included.

To be included in the meta-analysis on survival, articles had to provide a hazard ratio (HR) and a 95\% confidence interval (CI) or a beta coefficient and a standard error (SE) for the comparison of mortality risk between patients with and without hyponatremia. Only studies reporting a multivariate HR were included in order to reduce heterogeneity. As insignificant multivariate HRs were not reported in two studies $(15,16)$, the authors were contacted to obtain the data; however, neither of them responded. 
Consequently, the two studies were excluded from the meta-analysis.

\section{Data extraction and quality assessment}

Titles and abstracts were screened to identify eligible articles. The first 400 abstracts were randomly selected and screened by two authors (AWL and BSP) to validate inclusion and exclusion criteria. Any disagreement was resolved by consensus. The remaining titles and abstracts were screened by one author (AWL), and in any case of doubt, the abstract under consideration was assessed by both authors. After identification of likely eligible fulltext articles, 20 randomly selected articles were read and included/excluded by both authors. The remaining articles were included or excluded through full-text reading by one author (AWL), and in case of doubt, the article in question was discussed by the two authors until consensus was reached. Covidence (covidence.org) and Endnote (Clarivate Analytics) were used for reference management during the inclusion and exclusion procedure including duplicate screening. Data extraction was divided between the two authors (AWL and BSP) and subsequently checked by the other author. Following data was extracted from the studies: name of first author, publication year, country, rating, study design, sample size, study design, clinical characteristics of the study population, sodium level cut-off, follow-up time and risk estimate for overall survival (OS). Articles included in the study were quality scored by both authors using a modified version of the Quality of Prognosis Studies Tool (QUIPS) (17) and rated as high quality (+++), acceptable quality $(++)$ and low quality (+). The protocol was submitted to the PROSPERO database (ID 184612).

\section{Statistical analyses}

The primary outcome was OS and results from articles reporting adjusted risk estimates with CIs or SEs for mortality was combined in a meta-analysis. Heterogeneity between studies was assessed by using two statistical methods, Cochran $\mathrm{Q}$ and $\mathrm{I}^{2}(18)$. If data indicated the presence of heterogeneity $\left(\mathrm{P}<0.1\right.$ and/or $\left.\mathrm{I}^{2}>50 \%\right)$, the DerSimonian-Laird method for a random-effects model was used to calculate the pooled HRs. Otherwise was the fixedeffects model used. No subgroup analysis was performed owed to the limited number of studies. The possibility of publication bias was evaluated by a funnel plot, Begg's test and Egger's test. We did not pool results for other outcomes than OS because of the small number of studies. The robustness of the pooled result was evaluated by a sensitivity analysis omitting low quality studies. A P value $<0.05$ was considered significant. All statistical analyses were performed with Stata software version 15.1 (Stata Corporation, College Station, TX, USA), and all $\mathrm{P}$ values were two-sided.

\section{Results}

\section{Study selection}

The electronic database search identified 10,888 articles. After removal of duplicates, 6,890 articles were identified. Titles and abstracts were screened, and subsequent, 61 full-text articles were examined for eligibility. Of these, 15 articles meet the inclusion and exclusion criteria for the review. However, two studies $(12,19)$ included overlapping study populations, and one of these articles was thus excluded (19). Therefore, a total of 14 articles were included in the systematic review. Only ten of these articles reported a useful risk estimate and were included in the meta-analysis. The inclusion and exclusion procedures are illustrated in Figure 1.

\section{Study description and quality assessment}

Descriptive data for the studies included in the systematic review are summarized in Table 1. All studies were retrospective cohort studies except for a single prospective study (20). The articles were published between 1998 and 2019. The studies included a total of 4,364 NSCLC patients with sample sizes in each paper ranging from 35 to 671 patients. Half of the articles (seven articles) $(13,15,16,22,23,26,27)$ originated from Asia, primarily India. The remaining seven articles originated from Europe (six articles) $(12,20,21,24,25,28)$ and Unites States (one article) (5). The median/mean age of included patients varied from 49 to 72 years. The majority of studies included only stage IIIb and IV patients $(12,13,15,20,22,24,26,28)$, but three studies included stage I and stage II patients as well as more advanced stage patients $(16,20,23)$. The patients' disease stage was not reported in two studies $(5,21)$. Staging procedure was only reported in two studies $(15,22)$. Smoking history was reported in eight studies $(4,12,15,16,24,25,27,28)$ and the frequency of current and former smokers raged from $60 \%$ to $84 \%$.

The cut-off values used for defining hyponatremia varied 


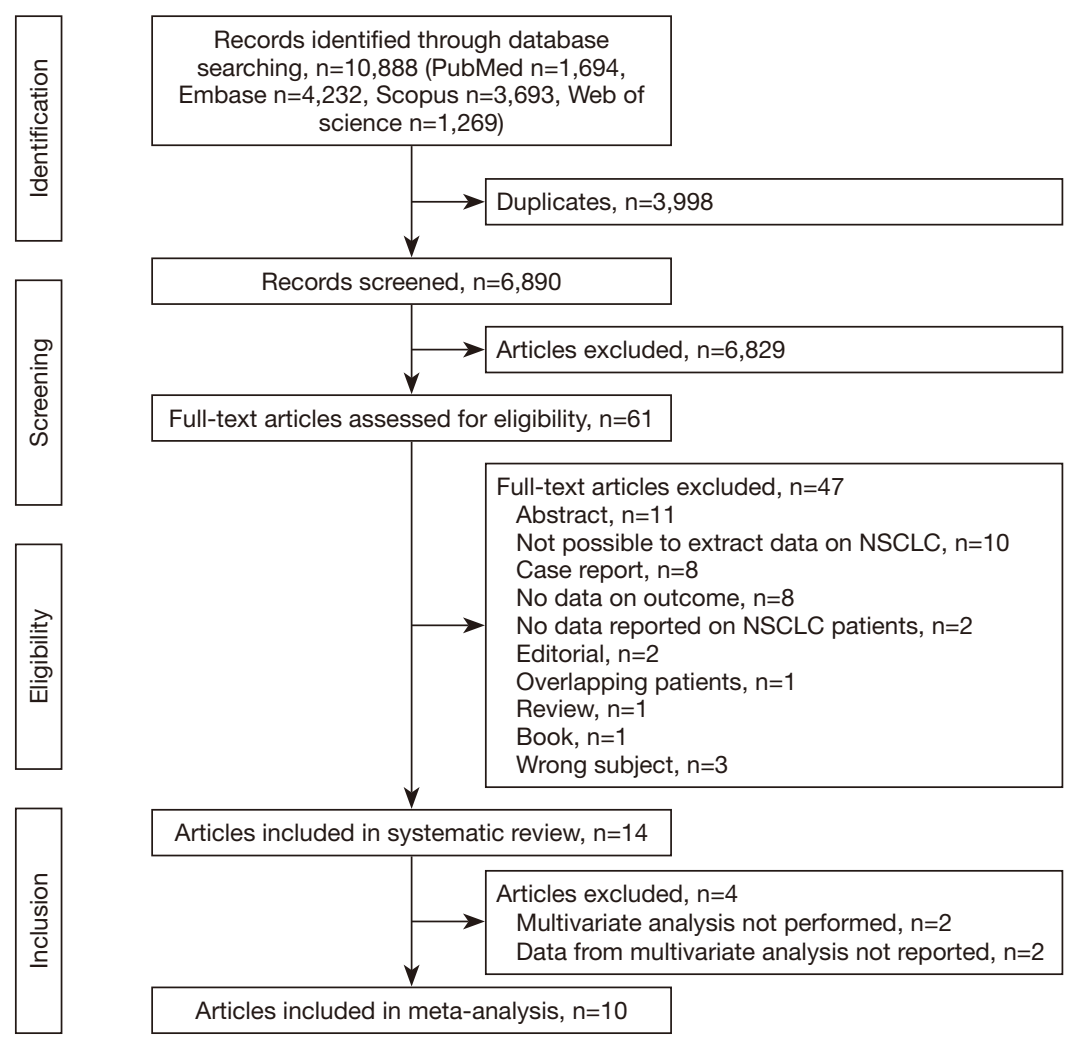

Figure 1 Flow diagram of the inclusion and exclusion procedures. NSCLC, non-small cell lung cancer.

significantly ranging from 132 to $141.9 \mathrm{mmol} / \mathrm{L}$, but the majority of studies used $135 \mathrm{mmol} / \mathrm{L}$ as a predefined cut-off (six studies) $(5,12,22,25,27,28)$. Three studies defined the optimal cut-off for the association by use of either receiver operating curve $(13,16)$ or the maximum log-rank statistical value (23). These studies all used high sodium levels as cutoffs $(139,140$ and $141.9 \mathrm{mmol} / \mathrm{L})$. The cut-off was not reported in one study (15). None of the studies reported any data on the underlying causes of the hyponatremia in the patients.

All studies included a mixed cohort of hyponatremic and eunatremic patients, and, using the defined cut-offs, a total of 1,534 patients were classified with hyponatremia in the studies. The frequency of hyponatremic patients was reported in 12 of the 14 studies $(5,12,16,20-28)$ and varied significantly ranging from $13 \%$ to $96 \%$ with an average of $39 \%$. Follow-up time was reported in five studies $(5,12,15,16,23)$ and the average follow-up time in these studies ranged from 12.6 months to 3.1 years. The overall quality of the studies was rated from low to high quality with six studies rating low quality $(5,16,22,23,26,28)$, seven rating acceptable $(13,15,20,21,24,25,27)$ and one study rating high quality (12).

\section{Hyponatremia and $O S$}

Of the 14 articles included in the systematic review, 10 articles reported an adjusted risk estimate of mortality risk between patients with and without hyponatremia and were thus included in the meta-analysis. Study characteristics, rating and adjustment variables for each of these studies are listed in Table 1. The studies were rated from low to high quality. Adjustment for stage and PS was performed in seven of the studies $(12,20,21,24,25,27,28)$ while six studies adjusted for sex $(20,21,24-26,28)$ and five studies adjusted for age and histology $(21,24-26,28)$. In one study (5), no information was reported on adjustment factors.

Because of substantial between-study heterogeneity $\left(\mathrm{Q}=32.14\right.$ on $\left.9 \mathrm{df} ; \mathrm{P}<0.001 ; \mathrm{I}^{2}=72 \% ; \mathrm{P}=0.0631\right)$, we calculated a pooled HR using a random-effects model. The pooled result showed that hyponatremia was associated with a $102 \%$ increased risk of death in patients with NCSLC [HR $=2.02$ (95\% CI: 1.65-2.47), Figure 2].

We observed indications of publication bias as the funnel 
Table 1 Studies investigating hyponatremia as a prognostic factor in NSCLC patients ( $\mathrm{n}=14)$

\begin{tabular}{|c|c|c|c|c|c|c|c|c|c|c|c|}
\hline $\begin{array}{l}\text { Author, year, country, } \\
\text { rating }\end{array}$ & $\begin{array}{l}\text { Design, study population, } \mathrm{n} \text {, } \\
\text { age (years) }\end{array}$ & Clinical stage & $\begin{array}{l}\text { Tumor histology, material for } \\
\text { diagnosis }\end{array}$ & $\begin{array}{l}\text { Smoking } \\
\text { history }\end{array}$ & Evaluation time & Treatment & $\begin{array}{l}\mathrm{Na}^{+} \text {cut- } \\
\text { off, } \\
\mathrm{mmol} / \mathrm{L}\end{array}$ & $\begin{array}{l}\text { Patients with } \\
\text { hypona', no./ } \\
\text { total no. }\end{array}$ & $\begin{array}{l}\text { Follow-up time/ } \\
\text { number of events }\end{array}$ & $\begin{array}{l}\text { Outcome, OS U/M, HR } \\
\text { (95\% Cl) or log-rank } \\
\text { P value }\end{array}$ & Adjustment variables \\
\hline $\begin{array}{l}\text { Rechnitzer, 1998, } \\
\text { Germany (20), } \\
\text { acceptable quality }\end{array}$ & $\begin{array}{l}\text { Prospective cohort, } \mathrm{n}=229 \\
\text { median age: } 57.4\end{array}$ & $\begin{array}{l}\text { I: } 1(0.4 \%) ; \text { II: } 6 \\
\text { (2.6\%); IIIA: } 20 \\
\text { (8.7\%); IIIB: } 64 \\
\text { (28.0\%); IV: } 132 \\
\text { (57.7\%); unknown: } \\
\text { 6 (2.6\%) }\end{array}$ & $\begin{array}{l}\text { Adeno: } 125 \text { (54.6\%); SCC: } \\
72(31.4 \%) ; \text { large cell: } 16 \\
\text { (7.0\%); mixed cell: } 16 \text { (7.0\%); } \\
\text { histologically or cytologically }\end{array}$ & NR & $\begin{array}{l}\text { At start of first-line } \\
\text { treatment }\end{array}$ & $\begin{array}{l}\text { Chemotherapy, RT } \\
\text { to primary tumor in } \\
\text { few cases }\end{array}$ & 137.5 & $59 / 229(26 \%)$ & $\begin{array}{l}220 \text { events }(96 \%) \\
\text { during follow-up. } \\
\text { Minimum follow-up } \\
\text { time of } 3.5 \text { years }\end{array}$ & $\begin{array}{l}\text { M: beta coefficient }=0.7736, \\
S E=0.1611\end{array}$ & Stage and sex \\
\hline $\begin{array}{l}\text { Jacot, 2001, France } \\
\text { (21), acceptable } \\
\text { quality }\end{array}$ & $\begin{array}{l}\text { Retrospective cohort, } \\
\mathrm{n}=231 \text {, median age: } 59 \\
{[32-85]}\end{array}$ & IV & $\begin{array}{l}\text { Adeno: } 86(37 \%) \text {; SCC: } 95(41 \%) ; \\
\text { large cell: } 50(22 \%) ; \text { histologically }\end{array}$ & NR & $\begin{array}{l}\text { At start of first-line } \\
\text { treatment }\end{array}$ & NA & 132 & $\begin{array}{l}219 / 229 \\
(96 \%)\end{array}$ & $\begin{array}{l}207 \text { death }(90 \%) \\
\text { during follow-up }\end{array}$ & M: $2.99(1.17-7.62)$ & $\begin{array}{l}\text { Sex, age, PS, histology, serum } \\
\text { NSE level, serum CYFRA 21-1 } \\
\text { level, serum albumin, alkaline } \\
\text { phosphatases, LDH, blood } \\
\text { leucocyte count, presence of } \\
\text { bone metastases, presence of } \\
\text { liver metastases, neurological } \\
\text { symptoms }\end{array}$ \\
\hline $\begin{array}{l}\text { Bose, 2011, India (22), } \\
\text { low quality }\end{array}$ & $\begin{array}{l}\text { Retrospective cohort, } \mathrm{n}=40 \text {, } \\
\text { mean age: } 49\end{array}$ & $\begin{array}{l}\text { IIIB: } 25(62 \%) ; \text { IV: } \\
\text { 15 (38\%) }\end{array}$ & $\begin{array}{l}\text { Adeno: } 16(40 \%) \text {; SCC: } 11 \text { (28\%); } \\
\text { large cell: } 3(7 \%) \text {; non-specified: } \\
10 \text { (25\%); FNAC or biopsy }\end{array}$ & NR & $\begin{array}{l}\text { At start of first-line } \\
\text { treatment }\end{array}$ & $\begin{array}{l}\text { Platinum-based } \\
\text { regimens }\end{array}$ & 135 & $7 / 35(20 \%)$ & $\begin{array}{l}27 \text { events (68\%) } \\
\text { during } 6 \text { months } \\
\text { follow-up }\end{array}$ & $\begin{array}{l}\text { U: log-rank } P<0.03 \text { (further } \\
\text { data NR) }\end{array}$ & - \\
\hline $\begin{array}{l}\text { Kobayashi, 2014, } \\
\text { Japan (23), low quality }\end{array}$ & $\begin{array}{l}\text { Retrospective cohort, } \\
\mathrm{n}=386 \text {, mean age: } 67.4, \mathrm{SD} \\
10.1\end{array}$ & 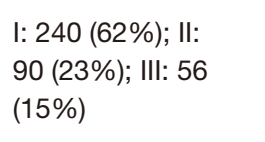 & $\begin{array}{l}\text { Adeno: } 241 \text { (62\%); SCC: } 96(25 \%) ; \\
\text { large: } 23(6 \%) \text {; other: } 26 \text { (7\%); } \\
\text { material NR }\end{array}$ & NR & Pre operation & NR & $139^{\ddagger}$ & $\begin{array}{l}123 / 386 \\
(32 \%)\end{array}$ & $\begin{array}{l}\text { Mean } 41.2 \text { months } \\
{[1-118]}\end{array}$ & M: $1.527(1.005-2.322)$ & $\begin{array}{l}\text { Tumor size, pleural invasion, } \\
\text { nodal status }\end{array}$ \\
\hline $\begin{array}{l}\text { Svaton, } 2014 \text {, } \\
\text { Czech Republic (24), } \\
\text { acceptable quality }\end{array}$ & $\begin{array}{l}\text { Retrospective cohort, } \\
\mathrm{n}=544, \text { median age: } 64 \\
{[28-84]}\end{array}$ & $\begin{array}{l}\text { IIIB: } 114 \text { (21.0\%); } \\
\text { IV: } 430 \text { (79.0\%) }\end{array}$ & $\begin{array}{l}\text { Adeno: } 255 \text { (46.9\%); SCC: } \\
260 \text { (47.8\%); NOS: } 29(5.3 \%) ; \\
\text { histologically or cytologically }\end{array}$ & $\begin{array}{l}\text { Current: } 254 \\
\text { (47\%); former: } \\
\text { 189 (35\%); } \\
\text { never: } 110 \\
(18 \%)\end{array}$ & $\begin{array}{l}\text { At start of erlotinib as } \\
\text { 2nd, 3rd, 4th or } 5 \text { th line } \\
\text { of treatment }\end{array}$ & Erlotinib & 136 & $\begin{array}{l}117 / 544 \\
(22 \%)\end{array}$ & NR & M: $1.87(1.47-2.39)$ & $\begin{array}{l}\text { Sex, age, smoking, histology, } \\
\text { stage, PS, treatment line, EGFR } \\
\text { status }\end{array}$ \\
\hline $\begin{array}{l}\text { Castillo, 2016, USA } \\
\text { (5), low quality }\end{array}$ & $\begin{array}{l}\text { Retrospective cohort, } \\
n=1,758 \text { (NSCLC } n=405), \\
\text { mean age: } 60.2, S D 13\end{array}$ & NA & NR & NR & $\begin{array}{l}\text { At cancer diagnosis and } \\
\text { during follow-up until } \\
\text { study end or death }\end{array}$ & NR & 135 & $\begin{array}{l}307 / 405 \\
(76 \%)\end{array}$ & $\begin{array}{l}\text { Total cohort: mean } 3.1 \\
\text { years (SD 2.7) }\end{array}$ & M: $2.8(2.0-3.9)$ & NR \\
\hline $\begin{array}{l}\text { Minami, 2016, Japan } \\
\text { (13), acceptable } \\
\text { quality }\end{array}$ & $\begin{array}{l}\text { Retrospective cohort, } \\
n=154 \text { ( } n=103 \text { evaluated), } \\
\text { median age: } 70 \text { [42-86] }\end{array}$ & $\begin{array}{l}\text { IIIB: } 41(40 \%) ; \mathrm{IV}: \\
62(60 \%)\end{array}$ & SCC; Histologically or cytologically & NR & $\begin{array}{l}\text { At start of first-line } \\
\text { treatment }\end{array}$ & Chemotherapy & $140^{\mathrm{s}}$ & NR & NR & U: $0.96(0.91-1.02)$ & - \\
\hline $\begin{array}{l}\text { Kogo, 2017, Japan } \\
\text { (15), acceptable } \\
\text { quality }\end{array}$ & $\begin{array}{l}\text { Retrospective cohort, } \\
\mathrm{n}=100, \text { median age: } 62 \\
{[32.5-80.7]}\end{array}$ & $\begin{array}{l}\text { IIIIb: 33 (33\%); IV: } \\
67(67 \%)\end{array}$ & $\begin{array}{l}\text { Adeno: } 73(73 \%) \text {; SCC: } 23(23 \%) \text {; } \\
\text { other: } 4(4 \%) ; \text { FNAC or brush } \\
\text { cytology }\end{array}$ & $\begin{array}{l}\text { Current/ } \\
\text { former: } 76 \\
(76 \%)\end{array}$ & $\begin{array}{l}\text { At start of first-line } \\
\text { treatment }\end{array}$ & $\begin{array}{l}\text { Platinum and } \\
\text { third-generation } \\
\text { chemotherapy }\end{array}$ & NR & NR & $\begin{array}{l}\text { Median } 12.6 \text { months } \\
(0.2-67.0)\end{array}$ & $\begin{array}{l}\text { U: } 0.909 \text { (0.829-0.996); M: } \\
\text { not significant (data NR) }\end{array}$ & NR \\
\hline $\begin{array}{l}\text { Berardi, 2017, Italy } \\
\text { (25), acceptable } \\
\text { quality }\end{array}$ & $\begin{array}{l}\text { Retrospective cohort, } \\
\mathrm{n}=433 \text {, median age: } 66 \\
{[25-86]}\end{array}$ & $\begin{array}{l}\text { III: } 112 \text { (26\%); IV: } \\
321(74 \%)\end{array}$ & $\begin{array}{l}\text { Adeno: } 278 \text { (64\%); SCC: } \\
101 \text { (23\%); other: } 54 \text { (13\%); } \\
\text { histologically or cytologically }\end{array}$ & $\begin{array}{l}\text { Former/ } \\
\text { current: } 364 \\
\text { (84\%); never: } \\
69(16 \%)\end{array}$ & $\begin{array}{l}\text { At start of first-line } \\
\text { treatment }\end{array}$ & $\begin{array}{l}\text { Platinum } \\
\text { chemotherapy: } 348 \\
\text { (80\%) } \\
\text { Non-platinum } \\
\text { based: } 57 \text { (13\%); } \\
\text { EGFR TKI: } 28 \text { (7\%) }\end{array}$ & 135 & $69 / 433(16 \%)$ & $\begin{array}{l}281 \text { events (65\%) } \\
\text { during follow-up }\end{array}$ & $\begin{array}{l}\text { U: } 1.71(1.25-2.34) ; \mathrm{M}: 1.59 \\
(1.14-2.21)\end{array}$ & $\begin{array}{l}\text { Sex, histology, stage, PS, EGFR } \\
\text { status }\end{array}$ \\
\hline $\begin{array}{l}\text { Doshi, } 2018 \text {, India } \\
\text { (26), low quality }\end{array}$ & $\begin{array}{l}\text { Retrospective cohort, } \\
\mathrm{n}=257, \text { median age: } 55 \\
{[21-78]}\end{array}$ & IIIlb/V & $\begin{array}{l}\text { Histology NR; histologically or } \\
\text { cytologically }\end{array}$ & $\begin{array}{l}\text { Current: } 116 \\
(45 \%) ; \text { never: } \\
82(31 \%)\end{array}$ & $\begin{array}{l}\text { At start of first-line } \\
\text { treatment }\end{array}$ & $\begin{array}{l}\text { Pemetrexed- } \\
\text { platinum doublet }\end{array}$ & 136 & $\begin{array}{l}137 / 257 \\
(53 \%)\end{array}$ & NR & M: $2.07(1.11-3.84)(n=83)$ & $\begin{array}{l}\text { Sex, age, smoking, PS, EGFR } \\
\text { status }\end{array}$ \\
\hline
\end{tabular}


Table 1 (continued)

\begin{tabular}{|c|c|c|c|c|c|c|c|c|c|c|c|}
\hline $\begin{array}{l}\text { Author, year, country, } \\
\text { rating }\end{array}$ & $\begin{array}{l}\text { Design, study population, } \mathrm{n} \text {, } \\
\text { age (years) }\end{array}$ & Clinical stage & $\begin{array}{l}\text { Tumor histology, material for } \\
\text { diagnosis }\end{array}$ & $\begin{array}{l}\text { Smoking } \\
\text { history }\end{array}$ & Evaluation time & Treatment & $\begin{array}{l}\mathrm{Na}^{+} \text {cut- } \\
\text { off, } \\
\mathrm{mmol} / \mathrm{L}\end{array}$ & $\begin{array}{l}\text { Patients with } \\
\text { hypona', no./ } \\
\text { total no. }\end{array}$ & $\begin{array}{l}\text { Follow-up time/ } \\
\text { number of events }\end{array}$ & $\begin{array}{l}\text { Outcome, OS U/M, HR } \\
(95 \% \mathrm{Cl} \text { ) or log-rank } \\
\text { P value }\end{array}$ & Adjustment variables \\
\hline $\begin{array}{l}\text { Lee, 2018, Korea (27), } \\
\text { acceptable quality }\end{array}$ & $\begin{array}{l}\text { Retrospective cohort, } \\
n=126, \text { age: }<75 \text { years: } 80 \\
(63.5 \%), 275 \text { years: } \\
46(36.5 \%)\end{array}$ & IV & $\begin{array}{l}\text { Non-SCC: } 63 \text { (50\%); SCC: } 63 \\
(50 \%) ; \text { material NR }\end{array}$ & $\begin{array}{l}\text { Former/ } \\
\text { current: } 99 \\
\text { (79\%); never: } \\
27 \text { (21\%) }\end{array}$ & $\begin{array}{l}\text { At start of second-line } \\
\text { treatment }\end{array}$ & $\begin{array}{l}\text { EGFR TKI: } \\
52(41 \%) ; \\
\text { chemotherapy: } 74 \\
\text { (59\%) }\end{array}$ & 135 & $68 / 126(54 \%)$ & NR & M: 3.228 (1.685-6.186) & $\begin{array}{l}\text { PS, brain metastasis, PFS } \\
\text { of first-line treatment, serum } \\
\text { albumin, SCS, histology, stage, } \\
\text { subsequent treatment, BSA } \\
\text { change, plasma } \mathrm{Hb}\end{array}$ \\
\hline $\begin{array}{l}\text { Fucà, 2018, Italy (12), } \\
\text { high quality }\end{array}$ & $\begin{array}{l}\text { Retrospective cohort, } \\
\mathrm{n}=197, \text { age: <65: } 84(43 \%), \\
\geq 65: 113(57 \%)\end{array}$ & IV & $\begin{array}{l}\text { Non-SCC: } 152 \text { (77\%); SCC: } 45 \\
(23 \%) ; \text { material NR }\end{array}$ & $\begin{array}{l}\text { Current: } 53 \\
(29 \%) ; \text { never/ } \\
\text { former: } 127 \\
(71 \%)\end{array}$ & $\begin{array}{l}\text { At start of } 1 O \text { as any line } \\
\text { of treatment }\end{array}$ & $\begin{array}{l}\text { PD-1/PD-L1 } \\
\text { therapy as single } \\
\text { therapy (191, 97\%) } \\
\text { or combined with } \\
\text { CTLA-4 blockade } \\
(6,3 \%)\end{array}$ & 135 & 26/197 (13\%) & Median 25.7 months & $\begin{array}{l}\text { U: } 3.00 \text { (1.80-4.80); M: } 3.11 \\
(1.91-5.05)\end{array}$ & $\begin{array}{l}\text { PS, liver metastases, bone } \\
\text { metastases }\end{array}$ \\
\hline $\begin{array}{l}\text { Li, 2019, China (16), } \\
\text { low quality }\end{array}$ & $\begin{array}{l}\text { Retrospective cohort, } \\
\mathrm{n}=1,304(\mathrm{n}=671 \text { evaluated), } \\
\text { age: } \leq 65: 478,>65: 193\end{array}$ & $\begin{array}{l}\text { I: } 323(48 \%) ; \text { II: } \\
156(23 \%) ; \text { II + IV: } \\
192(29 \%)\end{array}$ & $\begin{array}{l}\text { Adeno: } 366 \text { (54\%); SCC: } 239 \\
\text { (36\%); others: } 66 \text { (10\%); material } \\
\text { NR }\end{array}$ & $\begin{array}{l}\text { Former/ } \\
\text { current: } 400 \\
(60 \%) ; \text { never: } \\
271(40 \%)\end{array}$ & Before surgery & NR & $141.9^{8}$ & $\begin{array}{l}165 / 671 \\
(25 \%)\end{array}$ & Mean 40.84 [1-92] & $\begin{array}{l}\text { U: } 0.606 \text { (0.430-0.854); M: } \\
\text { not significant (data NR) }\end{array}$ & NR \\
\hline $\begin{array}{l}\text { Rinaldi, 2019, Italy/UK } \\
\text { (28), Iow quality }\end{array}$ & $\begin{array}{l}\text { Retrospective cohort, } \\
n=647, \text { median age: } 72 \\
{[32-93]}\end{array}$ & $\begin{array}{l}\text { IIIIB, IV, and IIIA } \\
\text { not suitable for } \\
\text { surgery }\end{array}$ & $\begin{array}{l}\text { Adeno: } 414 \text { (64\%); SCC: } \\
155 \text { (24\%); other: } 78(12 \%) ; \\
\text { histologically or cytologically }\end{array}$ & $\begin{array}{l}\text { Former/ } \\
\text { current: } 537 \\
\text { (83\%); never: } \\
110 \text { (17\%) }\end{array}$ & $\begin{array}{l}\text { At start of first-line } \\
\text { treatment }\end{array}$ & $\begin{array}{l}\text { Chemotherapy or } \\
\text { targeted therapy }\end{array}$ & 135 & $\begin{array}{l}237 / 647 \\
(37 \%)\end{array}$ & $\begin{array}{l}407 \text { events (63\%) } \\
\text { during follow-up }\end{array}$ & $\begin{array}{l}\text { U: } 1.36 \text { (1.12-1.71); M: } 1.29 \\
(1.03-1.54)\end{array}$ & $\begin{array}{l}\text { Sex, age, PS, smoking, stage, } \\
\text { histology, EGFR status, bone } \\
\text { metastasis }\end{array}$ \\
\hline
\end{tabular}

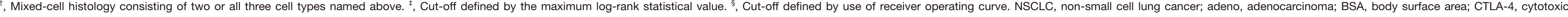

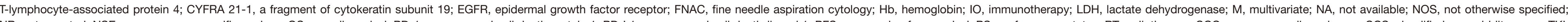

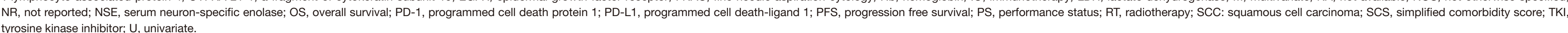




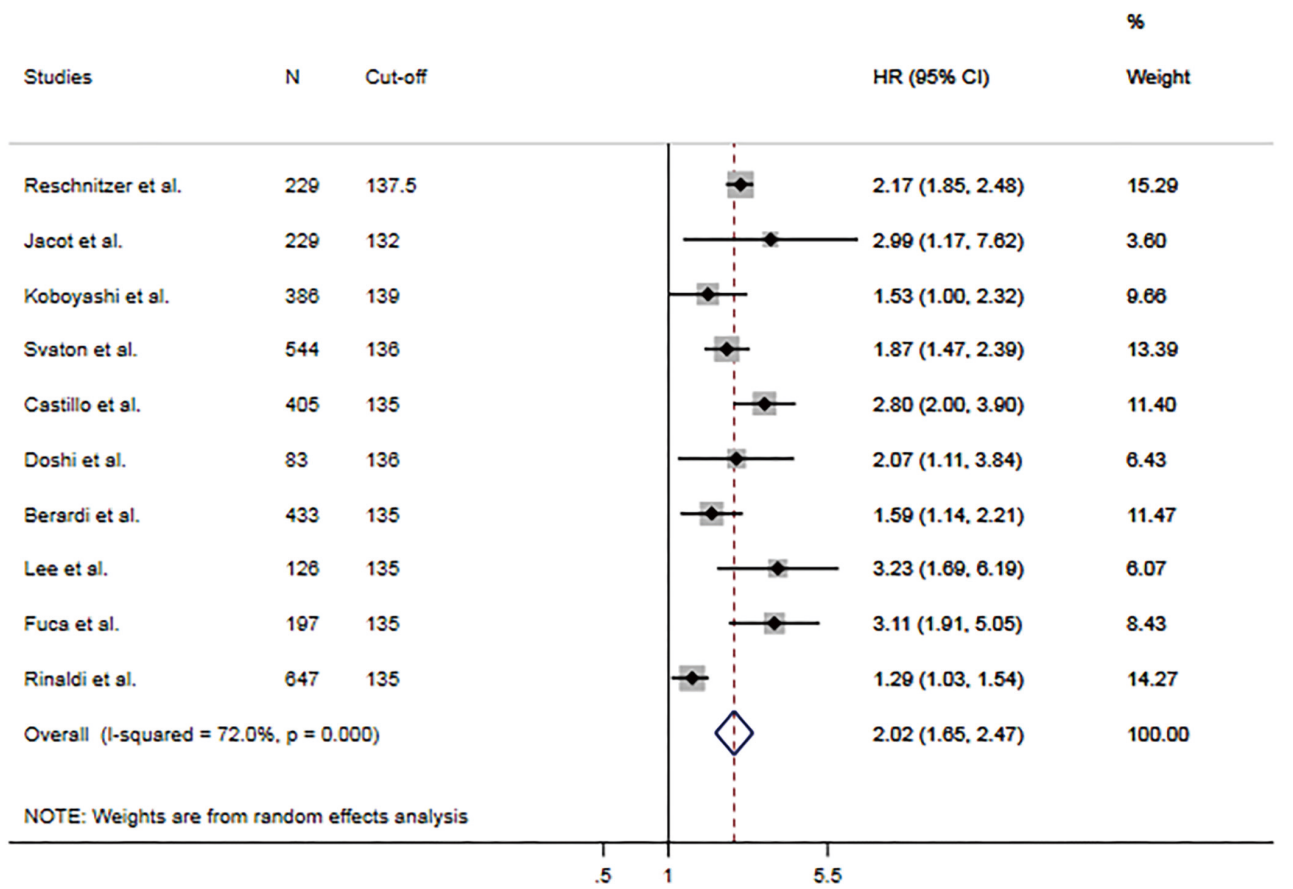

Figure 2 Forrest plot showing the HRs for the association of hyponatremia with OS in patients with NSCLC. HR, hazard ratio; OS, overall survival; NSCLC, non-small cell lung cancer; N, number of included patients; cut-off, the sodium cut-off level used in the study to define hyponatremia; CI, confidence interval.

plot visually showed asymmetry (Figure S1). Yet, there was no evidence of publication bias according to either Begg's test $(\mathrm{P}=0.531)$ nor Egger's test $(\mathrm{P}=0.442)$. In spite of this, a Trim and Fill analysis for OS was performed to impute missing studies in the funnel plot, and an adjusted pooled random-effect HR was calculated. The analysis showed a reduced pooled HR [1.84 (95\% CI: 1.51-2.23)], which however remained significant.

To assess the impact of the study quality, a sensitivity analyses were performed omitting the four low quality studies. Evaluating only the six studies of acceptable and high quality using a random-effects model, we found a pooled HR of 2.13 (95\% CI: 1.76-2.45).

\section{Hyponatremia and progression free survival (PFS)}

The correlation between hyponatremia and PFS was evaluated in four studies (12,24-26) and showed mixed results. Svaton et al. (24) found in a cohort of 544 patients treated with erlotinib as second, third, fourth or fifth line of treatment a significant increased risk of progression in patients with hyponatremia after adjusting for sex, age, smoking, histology, stage, PS, line of treatment and epidermal growth factor receptor status [adjusted HR $=1.25$ (95\% CI: 1.01-1.55)]. Fucà et al. (12) evaluated 197 patients treated with immunotherapy as any line of treatment and did not find a significant correlation between hyponatremia and PFS when adjusting for PD-L1 status, liver and bone metastases. Lastly, two studies evaluated patients treated with chemotherapy as first-line of treatment $(25,26)$. One study (26) found a significant shorter median PFS in hyponatremia patients but did not perform any multivariate analysis adjusting for potential confounders. No correlation was found in the other study (25).

\section{Sodium level normalization and outcome}

One study evaluated the association between sodium level normalization and PFS as well as OS in 69 stage III and IV patients with hyponatremia at start of first-line chemotherapy or targeted treatment (25). The sodium level was assessed after each treatment cycle. A significant association between normalization of sodium level and longer PFS and OS was reported. 


\section{Discussion}

This systematic review and meta-analysis indicates that hyponatremia is a relative common condition in NSCLC patients. Moreover, it was found to be associated with an increased mortality as a more than $100 \%$ increase in mortality risk was observed in hyponatremic patients compared with eunatremic patients. This finding was consistent after accounting for a possible publication bias; even though, the pooled HR was reduced. The majority of studies were retrospective studies and the overall quality of the available studies was low. Yet, the association was unchanged after omitting low quality studies from the analysis. Despite these concerns, for the first time this review collects the available literature and evaluates the association in NCSLC patients.

Hyponatremia is a common electrolyte abnormality (29) which has been associated with an increased mortality in several conditions as myocardial infarction (30), hepatic cirrhosis (31) and pulmonary embolism (32). In cancer patients, hyponatremia is mainly coursed by the syndrome of inappropriate antidiuretic hormone secretion (SIADH) which may arise due to ectopic secretion of arginine vasopressin (33). Yet, hyponatremia can also develop owing to several other conditions leading to loss of sodium or excess of water in relation to sodium as vomiting, diarrhea, chronic kidney disease, treatment with anticancer therapy and drugs used for palliation (34); all conditions regularly seen in cancer patients. In lung cancer, attention on hyponatremia has been focused on SCLC patients as SIADH is often observed in these patients (34). However, increasingly data has indicated that hyponatremia is additionally a frequent condition in NSCLC patients (5). The frequency of hyponatremia varied significantly in the studies included in this review with frequencies ranging from $13 \%$ to $96 \%$. The discrepancy could potentially be explained by different ways of reporting the incidence or different cut-offs. However, only one study evaluated hyponatremia at any time during follow-up, and thus found a high incidence of $76 \%$ (5), while the majority of studies $(13,15,16,20-23,25,26,28)$ estimated the frequency at time of diagnosis or before second of later lines of treatment $(12,24,27)$. Likewise, no clear correlation between high cut-offs for defining hyponatremia and incidence could be found as the three studies with highest cut-offs of $137.5 \mathrm{mmol} / \mathrm{L}$ (20), $139 \mathrm{mmol} / \mathrm{L} \mathrm{(23)} \mathrm{and}$ $141.5 \mathrm{mmol} / \mathrm{L}$ (16) found relatively low incidences of $26 \%, 32 \%$ and $25 \%$, respectively. Nevertheless, despite these substantial differences, data clearly indicates that hyponatremia is a common condition in NSCLC patients and needs attention in the daily clinic. As none of the included studies reported any data on the underlying causes of the hyponatremia in the patients, no evaluation of the causes of hyponatremia in NSCLC could be performed.

A meta-analysis is a useful tool to integrate results from individual studies in order to detect effects that may be missed by individual studies. In this meta-analysis, ten studies were found eligibly including a total of 3,279 NSCLC patients. The combined result demonstrates that hyponatremia is associated with a significantly increased mortality in NSCLC patients with a pooled HR of 2.02 (95\% CI: 1.65-2.47), which remained significant in a sensitivity analysis omitting low quality studies. Yet, in planning our meta-analysis, we chose only to include studies reporting an estimate of the adjusted HR to reduce the heterogeneity of the included studies. This caused omission of two studies with insignificant multivariate HRs as these estimates were not reported in the articles. This has undoubtedly led to an overestimation of our estimate. Moreover, we found indications of publication bias in the Funnel plot which can have influenced our estimate. However, our estimate remained significant after using the Trim and Fill analysis. Thus, we believe that the found association is reliable; even though, it may be of a reduced magnitude. Our meta-analysis is the first performed in NSCLC patients, but our finding is in line with a large number of previous studies conducted in cancer patient with other cancer types $(4,7,8,10,33,35,36)$ as well as several meta-analyses performed in other patients groups including patients with stroke (37), acute coronary syndrome (30) and chronic kidney disease (38), which all reports higher risk of mortality in hyponatremic patients. Our result thereby adds data to the existing literature highlighting that an increased focus on this electrolyte abnormality is highly important in NSCLC patients as well.

One study evaluated the value of normalization of sodium level during treatment. They found a significant association between normalized levels and prolonged PFS as well as OS in 69 patients with advanced stage disease. This finding is in line with data from a previous meta-analysis (39) addressing the effect of hyponatremia improvement on mortality in patients with diverse clinical conditions where any improvement of hyponatremia was associated with a reduced risk of overall mortality. One of the included studies comprised 61 patients with SCLC and supported this conclusion (10). However, data is sparse 
in lung cancer patients and more data is needed before drawing any firm conclusion.

The strengths of this study are the comprehensive systematic review of the available literature on hyponatremia as a prognostic factor in NSCLC patients and that each article was reviewed by two team members. We used four databases, applied broad search terms and used broad inand exclusion criteria in order not to miss relevant studies. Nonetheless, the study has some limitations to consider. Firstly, all studies, except for one study, were retrospective cohort studies, and the quality of the studies was overall low. Moreover, there was found substantial heterogeneity between the included studies and despite our attempt to manage this heterogeneity with appropriate meta-analytic techniques (e.g., random-effects model), studies varied in the cut-offs used for defining hyponatremia, the disease stages of the included patients, length of follow-up, and adjustment for confounding variables. Lastly, we excluded studies in language other than English, Danish, Swedish and Norwegian, and thus a language bias cannot be excluded.

\section{Conclusions}

This first systematic review and meta-analysis performed to evaluate hyponatremia as a prognostic factor in NSCLC patients indicates that hyponatremia is a relative common condition in NSCLC patients associated with an increased mortality. Hence, sodium level could be useful for stratifying NSCLC patients and, thereby, for preparing individual treatment plans and is thus a promising biomarker for use in the clinical management of this patient group.

\section{Acknowledgments}

Funding: None.

\section{Footnote}

Reporting Checklist: The authors have completed the PRISMA reporting checklist. Available at http://dx.doi. org/10.21037/tlcr-20-877

Peer Review File: Available at http://dx.doi.org/10.21037/ tlcr-20-877

Conflicts of Interest: All authors have completed the ICMJE uniform disclosure form (available at http://dx.doi. org/10.21037/tlcr-20-877). The authors have no conflicts of interest to declare.

Ethical Statement: The authors are accountable for all aspects of the work in ensuring that questions related to the accuracy or integrity of any part of the work are appropriately investigated and resolved.

Open Access Statement: This is an Open Access article distributed in accordance with the Creative Commons Attribution-NonCommercial-NoDerivs 4.0 International License (CC BY-NC-ND 4.0), which permits the noncommercial replication and distribution of the article with the strict proviso that no changes or edits are made and the original work is properly cited (including links to both the formal publication through the relevant DOI and the license). See: https://creativecommons.org/licenses/by-nc-nd/4.0/.

\section{References}

1. Torre LA, Bray F, Siegel RL, et al. Global cancer statistics, 2012. CA Cancer J Clin 2015;65:87-108.

2. Hirsch FR, Scagliotti GV, Mulshine JL, et al. Lung cancer: current therapies and new targeted treatments. Lancet 2017;389:299-311.

3. Chansky K, Sculier JP, Crowley JJ, et al. The International Association for the Study of Lung Cancer Staging Project: prognostic factors and pathologic TNM stage in surgically managed non-small cell lung cancer. Chinese Journal of Lung Cancer 2010;13:9-18.

4. Doshi SM, Shah P, Lei X, et al. Hyponatremia in hospitalized cancer patients and its impact on clinical outcomes. Am J Kidney Dis 2012;59:222-8.

5. Castillo JJ, Glezerman IG, Boklage SH, et al. The occurrence of hyponatremia and its importance as a prognostic factor in a cross-section of cancer patients. BMC Cancer 2016;16:564.

6. Berardi R, Rinaldi S, Caramanti M, et al. Hyponatremia in cancer patients: Time for a new approach. Crit Rev Oncol Hematol 2016;102:15-25.

7. Jeppesen AN, Jensen HK, Donskov F, et al. Hyponatremia as a prognostic and predictive factor in metastatic renal cell carcinoma. Br J Cancer 2010;102:867-72.

8. Kim HS, Yi SY, Jun HJ, et al. Clinical outcome of gastric cancer patients with bone marrow metastases. Oncology 2007;73:192-7.

9. Lassen U, Østerlind K, Hansen M, et al. Longterm survival in small-cell lung cancer: Posttreatment characteristics in patients surviving 5 to $18+$ years - an 
analysis of 1,714 consecutive patients. J Clin Oncol 1995;13:1215-20.

10. Hansen O, Sørensen P, Hansen KH. The occurrence of hyponatremia in SCLC and the influence on prognosis: a retrospective study of 453 patients treated in a single institution in a 10 -year period. Lung Cancer 2010;68:111-4.

11. Tiseo M, Buti S, Boni L, et al. Prognostic role of hyponatremia in 564 small cell lung cancer patients treated with topotecan. Lung Cancer 2014;86:91-5.

12. Fucà G, Galli G, Poggi M, et al. Low Baseline serum sodium concentration is associated with poor clinical outcomes in metastatic non-small cell lung cancer patients treated with immunotherapy. Target Oncol 2018;13:795-800.

13. Minami S, Ogata Y, Ihara S, et al. Outcomes and prognostic factors of chemotherapy for patients with locally advanced or metastatic pulmonary squamous cell carcinoma. Lung Cancer (Auckl) 2016;7:99-110.

14. Liberati A, Altman DG, Tetzlaff J, et al. The PRISMA statement for reporting systematic reviews and metaanalyses of studies that evaluate health care interventions: explanation and elaboration. PLoS Med 2009;6:e1000100.

15. Kogo M, Sunaga T, Nakamura S, et al. Prognostic index for survival in patients with advanced non-smallcell lung cancer treated with third-generation agents. Chemotherapy 2017;62:239-45.

16. Li W, Chen X, Wang L, et al. The prognostic effects of hyponatremia and hyperchloremia on postoperative NSCLC patients. Curr Probl Cancer 2019;43:402-10.

17. Hayden JA, van der Windt DA, Cartwright JL, et al. Assessing bias in studies of prognostic factors. Ann Intern Med 2013;158:280-6.

18. Higgins JPT, Thompson SG, Deeks JJ, et al. Measuring inconsistency in meta-analyses. BMJ 2003;327:557-60.

19. Fucà G, Mariani L, Lo Vullo S, et al. Weighing the prognostic role of hyponatremia in hospitalized patients with metastatic solid tumors: the HYPNOSIS study. Sci Rep 2019;9:12993.

20. Rechnitzer S, Bülzebruck H, Ebert W, et al. Pretreatment prognostic factors for survival in non-small-cell lung cancer: a multivariate analysis of 229 patients. Onkologie 1998;21:204-10.

21. Jacot W, Quantin X, Boher JM, et al. Brain metastases at the time of presentation of non-small cell lung cancer: a multi-centric AERIO analysis of prognostic factors. Br J Cancer 2001;84:903-9.

22. Bose CK, Dey S, Mukhopadhyay A. Hyponatremia of non-small cell lung cancer: Indian experience. Indian J Med Paediatr Oncol 2011;32:139-42.

23. Kobayashi N, Usui S, Yamaoka M, et al. The influence of serum sodium concentration on prognosis in resected non-small cell lung cancer. Thorac Cardiovasc Surg 2014;62:338-43.

24. Svaton M, Fiala O, Pesek M, et al. Predictive and prognostic significance of sodium levels in patients with NSCLC treated by Erlotinib. Anticancer Res 2014;34:7461-5.

25. Berardi R, Santoni M, Newsom-Davis T, et al. Hyponatremia normalization as an independent prognostic factor in patients with advanced non-small cell lung cancer treated with first-line therapy. Oncotarget 2017;8:23871-9.

26. Doshi KH, Shriyan B, Nookala MK, et al. Prognostic significance of pretreatment sodium levels in patients of nonsmall cell lung cancer treated with pemetrexedplatinum doublet chemotherapy. J Cancer Res Ther 2018;14:1049-53.

27. Lee SY, Kang EJ, Lee SY, et al. Efficacy of second-line treatment and importance of comorbidity scores and clinical parameters affecting prognosis in elderly patients with non-small cell lung cancer without epidermal growth factor receptor mutations. Oncol Lett 2018;15:600-9.

28. Rinaldi S, Santoni M, Leoni G, et al. The prognostic and predictive role of hyponatremia in patients with advanced non-small cell lung cancer (NSCLC) with bone metastases. Support Care Cancer 2019;27:1255-61.

29. Upadhyay A, Jaber BL, Madias NE. Incidence and prevalence of hyponatremia. Am J Med 2006;119:S30-5.

30. Ma QQ, Fan X De, Li T, et al. Short- and long-term prognostic value of hyponatremia in patients with acute coronary syndrome: a systematic review and metaanalysis. PLoS One 2018;13:e0193857.

31. Enescu A, Petrescu F, Mitruţ P, et al. Hyponatremia unfavourable prognostic factor in hepatic cirrhosis. Rom J Intern Med 2016;54:207-10.

32. Zhou XY, Chen HL, Ni SS. Hyponatremia and short-term prognosis of patients with acute pulmonary embolism: A meta-analysis. Int J Cardiol 2017;227:251-6.

33. Sørensen JB, Andersen MK, Hansen HH. Syndrome of inappropriate secretion of antidiuretic hormone (SIADH) in malignant disease. J Intern Med 1995;238:97-110.

34. Kitchlu A, Rosner MH. Hyponatremia in patients with cancer. Curr Opin Nephrol Hypertens 2019;28:433-40.

35. Aggerholm-Pedersen N, Rasmussen P, Dybdahl H, et al. Serum natrium determines outcome of treatment of advanced GIST with imatinib: a retrospective study 
of 80 patients from a single institution. ISRN Oncol 2011;2011:523915.

36. Allan SG, Stewart ME, Love S, et al. Prognosis at presentation of small cell carcinoma of the lung. Eur J Cancer 1990;26:703-5.

37. Chen Z, Jia Q, Liu C. Association of hyponatremia and risk of short- and long-term mortality in patients with stroke: a systematic review and meta-analysis. J Stroke Cerebrovasc Dis 2019;28:1674-83.

Cite this article as: Sandfeld-Paulsen B, Aggerholm-Pedersen N, Winther-Larsen A. Hyponatremia as a prognostic factor in nonsmall cell lung cancer: a systematic review and meta-analysis. Transl Lung Cancer Res 2021;10(2):651-661. doi: 10.21037/tlcr20-877
38. Sun L, Hou Y, Xiao Q, et al. Association of serum sodium and risk of all-cause mortality in patients with chronic kidney disease: a meta-analysis and sysematic review. Sci Rep 2017;7:15949.

39. Corona G, Giuliani C, Verbalis JG, et al. Hyponatremia improvement is associated with a reduced risk of mortality: evidence from a meta-analysis. PLoS One 2015;10:e0124105. 


\section{Supplementary}

The funnel plot of the meta-analysis evaluating the impact of hyponatremia on overall survival in patients with non-small cell lunge cancer. HR, hazard ratio

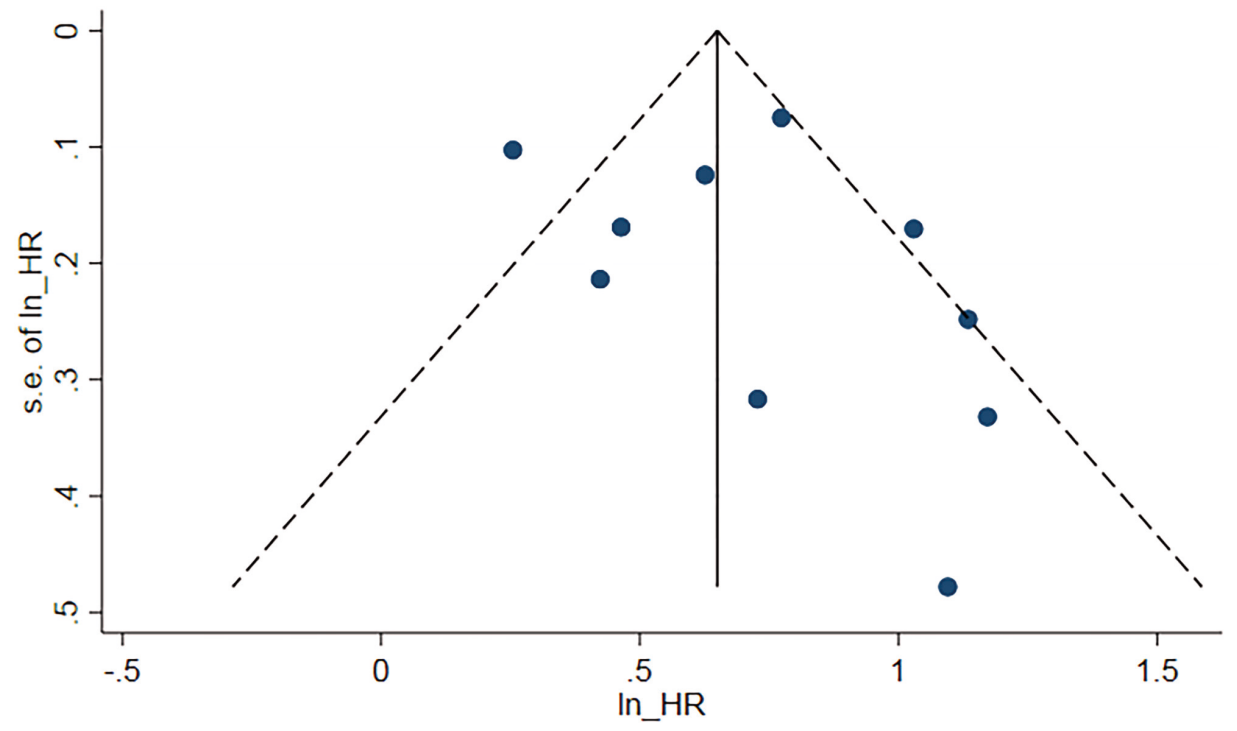

Figure S1 The funnel plot of the meta-analysis of the impact of hyponatremia on OS in patients with NSCLC. OS, overall survival; NSCLC, non-small cell lung cancer; HR, hazard ratio. 